\title{
REDUCTIONS OF TENSOR CATEGORIES MODULO PRIMES
}

\author{
PAVEL ETINGOF AND SHLOMO GELAKI \\ Dedicated to Miriam Cohen
}

\begin{abstract}
We study good (i.e., semisimple) reductions of semisimple rigid tensor categories modulo primes. A prime $p$ is called good for a semisimple rigid tensor category $\mathcal{C}$ if such a reduction exists (otherwise, it is called bad). It is clear that a good prime must be relatively prime to the Müger squared norm $|V|^{2}$ of any simple object $V$ of $\mathcal{C}$. We show, using the Ito-Michler theorem in finite group theory, that for group-theoretical fusion categories, the converse is true. While the converse is false for general fusion categories, we obtain results about good and bad primes for many known fusion categories (e.g., for Verlinde categories). We also state some questions and conjectures regarding good and bad primes.
\end{abstract}

\section{INTRODUCTION}

In this paper we study good (i.e., semisimple) reductions of semisimple rigid tensor categories (in particular, fusion categories) modulo primes. Namely, let $\mathcal{C}$ be a semisimple rigid tensor category over $\overline{\mathbb{Q}}$ (recall that any fusion category over a field of characteristic zero is defined over $\overline{\mathbb{Q}}$ by the Ocneanu rigidity theorem, see [ENO1, Theorem $2.28]$ ) and let $p$ be a prime. We say that $p$ is a good prime for $\mathcal{C}$ if there is a semisimple rigid tensor category $\mathcal{C}_{p}$ over $\overline{\mathbb{F}_{p}}$ (with the same Grothendieck ring as $\mathcal{C}$ ) which admits a lift to a tensor category over $\overline{\mathbb{Q}_{p}}$ (in the sense of [ENO1, Section 9.2]) that becomes equivalent to $\mathcal{C}$ after extension of scalars from $\overline{\mathbb{Q}}$ to $\overline{\mathbb{Q}_{p}}$. In this case, the category $\mathcal{C}_{p}$ is called a good reduction of $\mathcal{C}$ modulo $p$. Otherwise, if $\mathcal{C}_{p}$ does not exist, we say that $p$ is a bad prime for $\mathcal{C}$.

It is not hard to show that for any fusion category $\mathcal{C}$, there is a finite set of bad primes. The goal of this paper is to find conditions

Date: March 5, 2022.

Key words and phrases. tensor categories, good prime, reduction modulo a prime. 
for a prime to be bad for $\mathcal{C}$, and determine all such primes for various examples of fusion categories.

The organization of the paper is as follows. In Section 2, we discuss general properties of good and bad primes. In Section 3 we determine the bad primes for group-theoretical categories, in particular for representation categories of finite groups, using the Ito-Michler theorem in finite group theory; namely, we show that a prime is bad if and only if it divides the dimension of a simple object. In Section 4, we discuss bad primes for the Verlinde categories (i.e., fusion categories coming from quantum groups at roots of unity). Finally, in Section 5 we discuss some questions and conjectures regarding good and bad primes.

Acknowledgments. We are very grateful to Noah Snyder for useful discussions, in particular for contributing Example 4.5. The research of the first author was partially supported by the NSF grant DMS-1000113. The second author was supported by The Israel Science Foundation (grant No. 317/09). Both authors were supported by BSF grant No. 2008164.

\section{GOOD AND BAD PRIMES}

Let $p$ be prime. Let $\overline{\mathbb{Z}_{p}}$ denote the ring of integers in the field $\overline{\mathbb{Q}_{p}}$ (the algebraic closure of $\mathbb{Q}_{p}$ ). Let $\mathfrak{m}$ be the maximal ideal in $\overline{\mathbb{Z}_{p}}$. Clearly, $\overline{\mathbb{Z}_{p}} / \mathfrak{m} \cong \overline{\mathbb{F}_{p}}$.

Definition 2.1. Let $\mathcal{C}$ be a semisimple rigid tensor category over $\overline{\mathbb{Q}}$. A good reduction of $\mathcal{C}$ modulo $p$ is a semisimple rigid tensor category $\mathcal{C}_{p}$ over $\overline{\mathbb{F}_{p}}$, categorifying the Grothendieck ring of $\mathcal{C}$, such that there is a lift of $\mathcal{C}_{p}$ to $\overline{\mathbb{Z}_{p}}$ (i.e., a semisimple rigid tensor category $\widetilde{\mathcal{C}}_{p}$ over $\overline{\mathbb{Z}_{p}}$ which yields $\mathcal{C}_{p}$ upon reduction by the maximal ideal $\mathfrak{m}$ ) and a tensor equivalence $\widetilde{\mathcal{C}}_{p} \otimes_{\overline{\mathbb{Z}_{p}}} \overline{\mathbb{Q}_{p}} \cong \mathcal{C} \otimes_{\overline{\mathbb{Q}}} \overline{\mathbb{Q}_{p}}$. If a good reduction of $\mathcal{C}$ exists, we will say that $p$ is a good prime for $\mathcal{C}$. Otherwise we will say that $p$ is a bad prime for $\mathcal{C}$.

Example 2.2. A pointed fusion category $\operatorname{Vec}_{G}^{\omega}$, where $G$ is a finite group and $\omega$ is a 3 -cocycle of $G$ with values in $\overline{\mathbb{Q}}^{\times}$, has a good reduction modulo any prime, since $\omega$ can be chosen to take values in roots of unity.

Remark 2.3. Note that two non-equivalent fusion categories can have equivalent good reductions modulo a prime $p$. E.g., for any $3-\operatorname{cocycle} \omega$ on $\mathbb{Z} / p \mathbb{Z}$, the category $\operatorname{Vec}_{\mathbb{Z} / p \mathbb{Z}}^{\omega}$ reduces to $\operatorname{Vec}_{\mathbb{Z} / p \mathbb{Z}}$ (with trivial cocycle) in characteristic $p$ (as $\omega$ can be chosen to take values in $p$-th roots of unity). This cannot happen, however, if the global dimension of either of these two categories is relatively prime to $p$ (see [ENO1, Theorem $9.6])$. 
The following proposition gives a necessary condition for a prime to be good. Recall (see e.g., [Mu, ENO1]) that for any simple object $V$ of $\mathcal{C}$, one can define its Müger's squared norm $|V|^{2}$, which is an algebraic integer. Also recall that two algebraic integers are called relatively prime if their norms (which are usual integers) are relatively prime.

Proposition 2.4. Let $\mathcal{C}$ be a semisimple rigid tensor category over $\overline{\mathbb{Q}}$. If $p$ is a good prime for $\mathcal{C}$ then $p$ must be relatively prime to $|V|^{2}$ for any simple object $V \in \mathcal{C}$.

Proof. If $p$ is not relatively prime to $|V|^{2}$ then $|V|^{2}$ would have to be zero in the reduction $\mathcal{C}_{p}$. But Müger's squared norm of any simple object of a semisimple rigid tensor category must be nonzero (see [Mu, ENO1]).

Remark 2.5. One may ask if the converse of Proposition 2.4 holds. It turns out that the answer is negative in general (see Example 4.5 below). However, in the next section we will prove that the answer is positive for group-theoretical categories.

Recall that a fusion category $\mathcal{C}$ is called pseudounitary if the Müger's squared norm $|V|^{2}$ of every simple object $V$ coincides with the square FPdim $(V)^{2}$ of its Frobenius-Perron dimension. Recall also that every weakly integral fusion category $\mathcal{C}$ (i.e., such that $\operatorname{FPdim}(\mathcal{C})$ is an integer) is pseudounitary.

Corollary 2.6. If $\mathcal{C}$ is a pseudounitary fusion category then any good prime $p$ for $\mathcal{C}$ is relatively prime to the Frobenius-Perron dimension $\operatorname{FPdim}(V)$ for any simple object $V \in \mathcal{C}$.

Proposition 2.7. For any fusion category $\mathcal{C}$, there are finitely many bad primes.

Proof. If we write the structure morphisms of $\mathcal{C}$ in some basis, there will be only finitely many primes in the denominator, and all the other primes are automatically good.

Remark 2.8. Note that Proposition 2.7 is not true for infinite semisimple rigid tensor categories. For example, if $\mathcal{C}$ is the category of representations of $\operatorname{SL}(2, \overline{\mathbb{Q}})$ then all primes are bad for $\mathcal{C}$, since Müger's squared norm of the $n$-dimensional representation $V_{n-1}$ of $\operatorname{SL}(2, \overline{\mathbb{Q}})$ is $n^{2}$.

Recall ([DGNO], [ENO2]) that a fusion category is called weakly group-theoretical if it is obtained by a chain of extensions and equivariantizations from the trivial category. 
Proposition 2.9. If $\mathcal{C}$ is a weakly group-theoretical category of FrobeniusPerron dimension $d$ then any bad prime $p$ for $\mathcal{C}$ divides $d$.

Proof. The proof is by induction on the length of the chain. The base of induction is clear, and the induction step follows from the following lemma.

Lemma 2.10. Let $\mathcal{D}$ be a fusion category of global dimension $n$, and let $G$ be a finite group. Let $\mathcal{C}$ be either a $G$-extension of $\mathcal{D}$, or a $G$-equivariantization of $\mathcal{D}$. Let $p$ be a prime, which is relatively prime to both $n$ and $|G|$. If $\mathcal{D}$ admits a good reduction modulo $p$, then so does $\mathcal{C}$.

Proof. Let $\mathcal{D}_{p}$ be a good reduction of $\mathcal{D}$ modulo $p$. Since $p$ is relatively prime to $n$, this is a non-degenerate fusion category (i.e., its global dimension $\neq 0$ ), so the lifting theory of [ENO1, Section 9], applies. In particular, it follows from the proof of [ENO1, Theorem 9.6] that the lifting map $\operatorname{Eq}\left(\mathcal{D}_{p}\right) \rightarrow \operatorname{Eq}(\mathcal{D})$ between the groups of tensor autoequivalences of $\mathcal{D}_{p}$ and $\mathcal{D}$, defined in [ENO1, Section 9], is an isomorphism (i.e., any auto-equivalence of $\mathcal{D}$ has a reduction modulo $p$ ). This implies that we have an isomorphism of the corresponding categorical groups $\operatorname{Eq}\left(\mathcal{D}_{p}\right) \rightarrow \underline{\operatorname{Eq}}(\mathcal{D})$ (see [ENO3, Section 4.5]). Since $G$-actions on a fusion category $\mathcal{E}$ correspond to homomorphisms $G \rightarrow \operatorname{Eq}(\mathcal{E})$, we find that $G$-actions on $\mathcal{D}$ and $\mathcal{D}_{p}$ (and hence the corresponding equivariantizations) are in bijection. Moreover, since $|G|$ is coprime to $p$, it is easy to see that all the $G$-equivariantizations of $\mathcal{D}_{p}$ are semisimple. Thus, any $G$-equivariantization of $\mathcal{D}$ has a good reduction modulo $p$ (which is the corresponding $G$-equivariantization of $\mathcal{D}_{p}$ ).

Also, we see that the lifting map defines an isomorphism of BrauerPicard groups $\operatorname{BrPic}\left(\mathcal{D}_{p}\right) \rightarrow \operatorname{BrPic}(\mathcal{D})$ (see [ENO3, Section 4.1]). This follows from the fact that by [ENO3, Theorem 1.1], for any fusion category $\mathcal{E}$ the Brauer-Picard group $\operatorname{BrPic}(\mathcal{E})$ is naturally isomorphic to the group $\operatorname{EqBr}(\mathcal{Z}(\mathcal{E}))$ of braided auto-equivalences of the Drinfeld center $\mathcal{Z}(\mathcal{E})$, and from the proof of [ENO1, Theorem 9.6]. This implies that we have an isomorphism of Brauer-Picard groupoids $\underline{\operatorname{BrPic}\left(\mathcal{D}_{p}\right)} \rightarrow$ $\operatorname{BrPic}(\mathcal{D})$ (see [ENO3, Section 4.1]). Since for any fusion category $\mathcal{E}$,

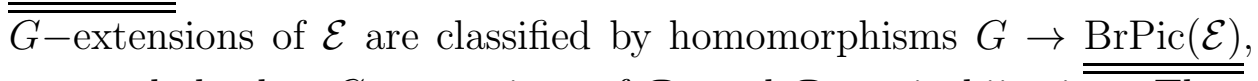
we conclude that $G$-extensions of $\mathcal{D}_{p}$ and $\mathcal{D}$ are in bijection. Thus, any $G$-extension of $\mathcal{D}$ has a good reduction modulo $p$ (which is the corresponding $G$-extension of $\mathcal{D}_{p}$ ).

The proposition is proved. 


\section{BAD PRIMES FOR GROUP-THEORETICAL CATEGORIES}

Let $G$ be a finite group and let $\omega \in Z^{3}\left(G, \overline{\mathbb{Q}}^{\times}\right)$be a normalized 3 -cocycle on $G$. Let $\operatorname{Vec}_{G}^{\omega}$ be the fusion category of finite-dimensional $G$-graded $\overline{\mathbb{Q}}$-vector spaces with the associativity defined by $\omega$. Let $H$ be a subgroup of $G$ such that $\left.\omega\right|_{H}$ is cohomologically trivial, and let $\psi \in C^{2}\left(H, \overline{\mathbb{Q}}^{\times}\right)$be such that $\left.\omega\right|_{H}=d \psi$; then the twisted group algebra $\overline{\mathbb{Q}}^{\psi}[H]$ is a unital associative algebra in $\operatorname{Vec}_{G}^{\omega}$. The grouptheoretical category $\mathcal{C}=\mathcal{C}(G, H, \omega, \psi)$ is defined as the fusion category of $\overline{\mathbb{Q}}^{\psi}[H]$-bimodules in $\operatorname{Vec}_{G}^{\omega}$ (see [ENO1, Definition 8.40], [0]).

Let $R$ be a set of representatives of double cosets of $H$ in $G$. In [O] it is explained that there is a bijection between the isomorphism classes of simple objects $V_{g, \rho}$ in $\mathcal{C}$ and isomorphism classes of pairs $(g, \rho)$, where $g \in R$ and $\rho$ is an irreducible projective representation of $H^{g}:=H \cap g \mathrm{Hg}^{-1}$ with a certain 2-cocycle $\psi^{g}$. (See also GN].) The dimension of $V_{g, \rho}$ is $\frac{|H|}{\left|H^{g}\right|} \operatorname{dim}(\rho)$.

Assume now that $H \subseteq G$ contains an abelian subgroup $A$ which is normal in $G$. Let $K:=H / A$, and assume that the orders of $K$ and $A$ are coprime (so that $H$ is isomorphic to $A \rtimes K$ ).

Proposition 3.1. Suppose that $\left.\psi^{g}\right|_{A}$ is cohomologically trivial for any $g \in G$. Then there exist cocycles $\eta \in Z^{2}\left(G / A, A^{\vee}\right), \widetilde{\omega} \in Z^{3}\left(\widetilde{G}_{\eta}, \overline{\mathbb{Q}}^{\times}\right)$ and a 2 - cochain $\widetilde{\psi} \in C^{2}\left(K, \overline{\mathbb{Q}}^{\times}\right)$such that $\mathcal{C} \cong \mathcal{C}\left(\widetilde{G}_{\eta}, K, \widetilde{\omega}, \widetilde{\psi}\right)$, where the crossed product $\widetilde{G}_{\eta}:=A^{\vee} \rtimes_{\eta} G / A$ is the extension of $G / A$ by $A^{\vee}$ using $\eta$.

Remark 3.2. Note that since the order of $K$ is relatively prime to the order of $A$, we have an embedding of $K$ into $\widetilde{G}_{\eta}$ defined canonically up to conjugation. In Proposition 3.1, we can use any such embedding.

Proof. In the special case $K=1$, this is [N, Theorem 4.9]. This theorem claims that there exist $\eta$ and $\widetilde{\omega}$ such that $\mathcal{C}(G, A, \omega, \psi)$ is equivalent to $\mathcal{C}\left(\widetilde{G}_{\eta},\{1\}, \widetilde{\omega}, 1\right)$. Now consider the module category $\mathcal{M}(H, \psi)$ over $\mathcal{C}(G, A, \omega, \psi)$. It is shown by a direct computation that under the above equivalence, this module category goes to a module category of the form $\mathcal{M}(K, \widetilde{\psi})$. Passing to the dual categories, we get the statement of the proposition.

Example 3.3. Consider the symmetric group on three letters $S_{3}$. Then $\operatorname{Rep}\left(S_{3}\right)=\mathcal{C}\left(S_{3}, S_{3}, 1,1\right)=\mathcal{C}\left(S_{3}, S_{2}, 1,1\right)$, which shows that 3 is a good prime for $\operatorname{Rep}\left(S_{3}\right)$.

We will use the following deep theorem, whose proof relies on the classification of finite simple groups, in an essential way. 
Theorem 3.4. (Ito-Michler, [Mi], see also [It]) Let $G$ be a finite group and let $p$ be a prime number dividing the order of $G$. Suppose that $p$ does not divide the dimension of any irreducible representation of $G$. Then the Sylow p-subgroup $S$ of $G$ is both normal and abelian, so (by a theorem of Schur) $G=S \rtimes K$, where the order of $K$ is not divisible by $p$.

We can now state and prove the main result of this section.

Theorem 3.5. Let $\mathcal{C}=\mathcal{C}(G, H, \omega, \psi)$ be a group-theoretical category. A prime number $p$ is bad for $\mathcal{C}$ if and only if $p$ divides the dimension of some simple object of $\mathcal{C}$.

Proof. The "if" direction follows from Proposition 2.4.

Let us prove the "only if" direction. Assume that $\omega$ and $\psi$ take values in roots of unity (which we can do without loss of generality). Take a prime $p$. If $p$ does not divide the order of $H$, the category $\mathcal{C}_{p}:=\mathcal{C}\left(G, H, \omega_{p}, \psi_{p}\right)$ (where $\omega_{p}, \psi_{p}$ are the reductions of $\omega, \psi$ modulo $p$ ) is semisimple over $\overline{\mathbb{F}}_{p}$, so $p$ is good for $\mathcal{C}$.

Therefore, it suffices to consider the case when $p$ divides the order of $H$. Suppose that $p$ does not divide the dimension of any simple object of $\mathcal{C}$, and let us show that $p$ is good. Since $\operatorname{Rep}(H)$ is a fusion subcategory of $\mathcal{C}$, Theorem 3.4 implies that $H=A \rtimes K$, where the order of $K$ is not divisible by $p$ and $A$ is an abelian normal $p$-subgroup of $H$.

We claim that in fact $A$ is normal in $G$, not only in $H$. Indeed, by our assumption, $p$ does not divide $|H| /\left|H^{g}\right|$, which implies that the order of $H^{g}$ is divisible by the order of $A$, so $H^{g}$ must contain $A$. So for any $g \in G$ we have $g^{-1} A g \subseteq A \rtimes K$, hence $g^{-1} A g=A$ (since $K$ is a $p^{\prime}$-group).

Finally, we claim that $\left.\psi^{g}\right|_{A}$ is trivial. Indeed, a projective representation of $A$ with 2-cocycle $\left.\psi^{g}\right|_{A}$ is a simple object of $\mathcal{C}$, so its dimension must be coprime to $p$. But the dimension of this object is a power of $p$, which is 1 only if $\psi^{g}$ is trivial, as desired.

Now, by Proposition 3.1, $\mathcal{C}$ is equivalent to $\mathcal{C}\left(\widetilde{G}_{\eta}, K, \widetilde{\omega}, \widetilde{\psi}\right)$. We can assume that $\widetilde{\omega}, \widetilde{\psi}$ take values in roots of unity. Since $p$ does not divide the order of $K$, the category $\mathcal{C}\left(\widetilde{G}_{\eta}, K, \widetilde{\omega}, \widetilde{\psi}\right)$ admits a reduction $\mathcal{C}\left(\widetilde{G}_{\eta_{p}}, K, \widetilde{\omega}_{p}, \widetilde{\psi}_{p}\right)$ to a fusion category in characteristic $p$, so $p$ is good for $\mathcal{C}$.

Corollary 3.6. Let $G$ be a finite group. A prime number $p$ is good for $\operatorname{Rep}(G)$ if and only if $p$ does not divide the dimension of any irreducible representation of $G$. 
Example 3.7. Corollary 3.6 fails for reductive algebraic groups. Indeed, consider the group $G:=\operatorname{PGL}(2, \overline{\mathbb{Q}})$, and let $\mathcal{C}:=\operatorname{Rep}(G)$ be the category of rational representations of $G$. Its simple objects $V_{2 m}$ have dimensions $2 m+1$, where $m$ is a nonnegative integer. Thus, the prime 2 is the only prime that has a chance to be good for this category, since any other prime divides the dimension (and hence, Müger's squared norm) of some irreducible object. Nevertheless, we claim that the prime 2 is bad for $\operatorname{Rep}(G)$ (so in fact, for this category, all primes are bad). Indeed, suppose we have a good reduction $\mathcal{C}_{2}$ of $\operatorname{Rep}(G) \bmod$ 2 , and let us derive a contradiction.

Let $X \in \mathcal{C}_{2}$ be the reduction of the 3 -dimensional (adjoint) representation of $G$. Then we have a unique morphism $m: X \otimes X \rightarrow X$ and isomorphism $b: X \rightarrow X^{*}$ up to scaling. Thus, if we fix the scaling of these, we can attach an amplitude $A(T)$ to any planar trivalent graph (allowing multiple edges but no self-loops), by contracting the morphisms $m$ corresponding to the vertices using $b$. Moreover, the amplitude $A\left(T_{2}\right)$ of the simplest planar trivalent graph $T_{2}$ (with 2 vertices and 3 edges, i.e., a circle with a diameter) is nonzero. Let us normalize it to be 1 . Then we get a well defined amplitude of any other planar trivalent graph.

Consider the amplitude $A\left(T_{4}\right)$ of the next simplest graph $T_{4}$ with 4 vertices - the square with diagonals (with one of the diagonals going outside the square to avoid intersection and realize the graph in the plane). It is supposed to be the reduction mod 2 of the corresponding amplitude $A_{\overline{\mathbb{Q}}}\left(T_{4}\right)$ over $\overline{\mathbb{Q}}$. But over $\overline{\mathbb{Q}}$, this is a computation in the representation theory of the Lie algebra $\mathfrak{s l}(2)$. Namely, if $x_{i}$ is an orthonormal basis of $\mathfrak{s l}(2)$, we get

$$
\left.\sum x_{i} x_{j} x_{i} x_{j}\right|_{V_{2}}=\left.A_{\overline{\mathbb{Q}}}\left(T_{4}\right)\left(\sum x_{i}^{2}\right)^{2}\right|_{V_{2}}
$$

where $V_{2}$ is the adjoint representation. A direct computation then shows that $A_{\overline{\mathbb{Q}}}\left(T_{4}\right)$ is $3 / 2$, which is a contradiction since $A\left(T_{4}\right)$ is supposed to be the reduction of this number modulo 2. 1

\footnotetext{
${ }^{1}$ For the reader's convenience, let us give explicit expressions of $A\left(T_{2}\right)$ and $A\left(T_{4}\right)$ in terms of $m$ and $b$. Let $m_{*}: X \rightarrow X \otimes X$ be the map obtained from the dual of $m$ by identifying $X^{*}$ with $X$ using $b$. Then we have

$$
A\left(T_{2}\right)=\operatorname{Tr}\left(m m_{*}\right), A\left(T_{4}\right)=\operatorname{Tr}\left(m(1 \otimes m)\left(m_{*} \otimes 1\right) m_{*}\right) .
$$

Using that if $X$ is the adjoint representation of $\mathfrak{s l}(2)$ then $m$ is the Lie bracket and $m_{*}$ is its dual under the Killing form, we arrive at formula (1).
} 


\section{VERLINDE CATEGORIES}

Let $\mathfrak{g}$ be a simple complex Lie algebra. For simplicity let us assume that it is simply laced (so $(\alpha, \alpha)=2$ for roots). Let $h$ be the Coxeter number of $\mathfrak{g}$, let $\theta$ be the highest root of $\mathfrak{g}$, and let $\rho$ be half the sum of positive roots of $\mathfrak{g}$.

Let $l>h$ be a positive integer, and let $q \in \mathbb{C}$ be such that the order of $q^{2}$ is $l$. Set $[n]_{q}:=\frac{q^{n}-q^{-n}}{q-q^{-1}}$.

Following Andersen and Paradowski AP, one can define the Verlinde fusion category $\mathcal{C}(\mathfrak{g}, q)$. Its simple objects are $V_{\lambda}$, where $\lambda$ are dominant weights for $\mathfrak{g}$ such that $(\lambda+\rho, \theta)<l$. The dimension of $V_{\lambda}$ is given by the $q$-Weyl formula:

$$
\operatorname{dim}\left(V_{\lambda}\right)=\prod_{\alpha>0} \frac{[(\lambda+\rho, \alpha)]_{q}}{[(\rho, \alpha)]_{q}}=q^{2(\lambda, \rho)} \prod_{\alpha>0} \frac{\left(1-q^{-2(\lambda+\rho, \alpha)}\right)}{\left(1-q^{-2(\rho, \alpha)}\right)} .
$$

We will need the following elementary and well known lemma (whose proof we include for the reader's convenience).

Lemma 4.1. Let $v$ be a primitive root of unity of order $n$. The norm $N(1-v)$ of $1-v$ is given by:

$$
N(1-v)=\left\{\begin{array}{l}
1, \quad n \text { is not a prime power } \\
p, \quad n \text { is a power of a prime } p
\end{array} .\right.
$$

Proof. Let $n=\prod_{i=1}^{m} p_{i}^{s_{i}}$ be the prime factorization of $n$. Then

$$
N(1-v)=\prod_{k=1, p_{1}, \ldots, p_{m} \nmid k}^{n}\left(1-v^{k}\right)
$$

which is the value of the cyclotomic polynomial $\prod_{k=1, p_{1}, \ldots, p_{m} \nmid k}^{n}\left(x-v^{k}\right)$ at $x=1$. By the exclusion-inclusion principle,

$$
\begin{aligned}
& \left.\prod_{k=1, p_{1}, \ldots, p_{m} \nmid k}^{n}\left(x-v^{k}\right)\right|_{x=1} \\
& =\left.\frac{\left(x^{n}-1\right) \prod_{i<j}\left(x^{\frac{n}{p_{i} p_{j}}}-1\right) \cdots}{\prod_{i}\left(x^{\frac{n}{p_{i}}}-1\right) \cdots}\right|_{x=1}=\frac{n \prod_{i<j} \frac{n}{p_{i} p_{j}} \cdots}{\prod_{i} \frac{n}{p_{i}} \cdots} .
\end{aligned}
$$

Now, the power of $p_{i}$ on the right hand side equals

$$
1-(m-1)+\left(\begin{array}{c}
m-1 \\
2
\end{array}\right)-\cdots=(1-1)^{m-1}=0
$$

unless $m=1$, in which case it equals 1 , as claimed. 
Theorem 4.2. Assume that $l$ is odd.

(i) If $l$ is a prime then any prime $p$ is good for $\mathcal{C}(\mathfrak{g}, q)$.

(ii) If $l$ is not a prime then a prime $p \geq h$ is $\operatorname{good}$ for $\mathcal{C}(\mathfrak{g}, q)$ if and only if $p$ does not divide $l$.

Remark 4.3. Note that the condition $p \geq h$ cannot be dropped in (ii). For instance, assume that $\mathfrak{g}=\mathfrak{s l}(n)($ so $h=n)$, and let $l=h+1$ (which we assume to be odd). Then $\mathcal{C}(\mathfrak{g}, q)$ is a pointed category, so it has no bad primes.

Proof. If $p$ is relatively prime to $l$, then one can define the fusion category $\mathcal{C}_{p}(\mathfrak{g}, q)$ over $\overline{\mathbb{F}_{p}}$ (similarly to $[\mathrm{AP}]$ ), so $p$ is a good prime.

(i) For $p=l$, there is a symmetric category over $\overline{\mathbb{F}_{p}}$ which lifts to the braided category $\mathcal{C}(\mathfrak{g}, q)$ (see [GM1, GM2, AP]), so again $p$ is good.

(ii) Suppose $p$ divides $l$. By Proposition 2.4, it is enough to show that at least one of the numbers $\operatorname{dim}\left(V_{\lambda}\right)$ is not relatively prime to $p$ or, equivalently, that its $p$-adic norm is $<1$. To this end, pick $\lambda:=\left(\frac{l}{p}-1\right) \rho$. Such $\lambda$ is allowed since $(\lambda+\rho, \theta)=\frac{(h-1) l}{p}<l$.

By Lemma 4.1, the $p$-adic norm of $1-v$ is 1 if the order of $v$ is not a prime power, and is $p^{-1 / p^{s}(p-1)}$ if the order of $v$ is equal to $p^{s+1}$. Now, the order of $q^{-2(\lambda+\rho, \alpha)}=q^{-2 l(\rho, \alpha) / p}$ is $p$ for any $\alpha$ (since by assumption, $(\rho, \alpha)<h \leq p)$. Thus, the $p$-adic norm of every factor in the numerator of the $q$-Weyl formula for $\operatorname{dim}\left(V_{\lambda}\right)$ is $p^{-1 /(p-1)}$, while the $p$-adic norm of every factor of the denominator is at least that, and the $p$-adic norm of $\left[\left(\rho, \alpha_{i}\right)\right]_{q}=1$ is 1 for any simple root $\alpha_{i}$. Thus, the $p$-adic norm of $\operatorname{dim}\left(V_{\lambda}\right)$ is $<1$, i.e., $\operatorname{dim}\left(V_{\lambda}\right)$ is not relatively prime to $p$, as desired.

Remark 4.4. If $l$ is a prime then the dimensions $\operatorname{dim}\left(V_{\lambda}\right)$ are units. Indeed, for any $1 \leq s<l$ there is a Galois automorphism sending $q^{2}$ to $q^{2 s}$, so the norm of $[s]_{q}=\frac{q^{s}-q^{-s}}{q-q^{-1}}$ is equal to 1 .

Example 4.5. The following example, due to Noah Snyder, shows that the converse to Proposition 2.4 fails for general (and even for braided) fusion categories. Indeed, consider the Verlinde category $\mathcal{C}\left(\mathfrak{s l}_{2}, q\right)$, where $q:=e^{\pi i / 8}$ (i.e., $l=8$ ), with simple objects $V_{0}=\mathbf{1}, V_{1}, \ldots, V_{6}$, and let $\mathcal{D}$ be its tensor subcategory generated by $V_{0}, V_{2}, V_{4}, V_{6}$. Then $\operatorname{dim}\left(V_{0}\right)=\operatorname{dim}\left(V_{6}\right)=1, \operatorname{dim}\left(V_{2}\right)=\operatorname{dim}\left(V_{4}\right)=1+\sqrt{2}$, which are all units, so any prime is relatively prime to the dimension of any simple object of $\mathcal{D}$. Yet, considering the graph $T_{4}$ as in Example 3.7, we get by a direct calculation

$$
A_{\overline{\mathbb{Q}}}\left(T_{4}\right)=\frac{[3]_{q}\left([3]_{q}-2\right)}{[3]_{q}-1}=\frac{1}{\sqrt{2}}
$$


which shows that 2 is a bad prime for $\mathcal{D}$.

Also, as was explained to us by Noah Snyder, it follows from the arguments similar to those in [MS] (computation of the "third twisted moment") that 3 is a bad prime for the 6-object Haagerup category, even though the dimensions of all its simple objects are units.2

\section{Conjectures And questions}

Question 5.1. If a prime $p$ is relatively prime to the global dimension $\operatorname{dim}(\mathcal{C})$, does $p$ have to be a good prime for $\mathcal{C}$ in cases (i)-(iii)?

(i) $\mathcal{C}$ is a general fusion category.

(ii) $\mathcal{C}$ is an integral fusion category, i.e., $\mathcal{C}=\operatorname{Rep}(H)$ for a semisimple quasi-Hopf algebra $H$.

(iii) $\mathcal{C}=\operatorname{Rep}(H)$ for a semisimple Hopf algebra $H$.

Note that in (ii) and (iii), $\operatorname{dim}(\mathcal{C})=\operatorname{dim}(H)$.

A positive answer in case (iii) would imply that any prime divisor of the dimension of a simple $H$-module divides the dimension of $H$, which is a weak (but still open) form of Kaplansky's 6-th conjecture.

Question 5.2. (i) Let $\mathcal{C}$ be an integral fusion category, and suppose that $p$ does not divide the (Frobenius-Perron) dimension of any simple object. Does $p$ have to be a good prime for $\mathcal{C}$, i.e, does Theorem 3.5 hold for $\mathcal{C}$ ?

(ii) Is this true for weakly group-theoretical categories?

Question 5.3. Does any fusion category $\mathcal{C}$ admit at most one good reduction modulo any prime $p$ ?

Remark 5.4. The answer is "yes" if $p$ is relatively prime to the global dimension of $\mathcal{C}$, by [ENO1, Theorem 9.6].

Question 5.5. Suppose that $p$ is a good prime for $\mathcal{C}$. Is $p \operatorname{good}$ for any module category over $\mathcal{C}$ ?

\section{REFERENCES}

[AP] H. H. Andersen and J. Paradowski, Fusion categories arising from semisimple Lie algebras, Comm. Math. Phys. Volume 169, Number 3 (1995), $563-588$.

[DGNO] V. Drinfeld, S. Gelaki, D. Nikshych, and V. Ostrik, On braided fusion categories I, Selecta Mathematica New Series, 16 (2010), 1-119. (A preliminary version: Group-theoretical properties of nilpotent modular categories, arXiv:0704.0195v2).

\footnotetext{
${ }^{2}$ As was explained to us by Noah Snyder, it can also be shown, using a specific presentation of the 6 -object Haagerup category (see [Iz), that any other prime is good for this category.
} 
[EG] P. Etingof and S. Gelaki, Some properties of finite-dimensional semisimple Hopf algebras, Math. Res. Lett. 5 (1998), no. 1-2, 191-197.

[ENO1] P. Etingof, D. Nikshych, V. Ostrik, On fusion categories, Ann. of Math. 162 (2005) 581-642.

[ENO2] P. Etingof, D. Nikshych and V. Ostrik, Weakly group-theoretical and solvable fusion categories, arXiv:0809.3031.

[ENO3] P. Etingof, D. Nikshych and V. Ostrik, Fusion categories and homotopy theory, Quantum topology, Volume 1, Issue 3, 2010, pp. 209-273.

[EO] P. Etingof and V. Ostrik, Finite tensor categories, Moscow Mathematical Journal 4 (2004), no. 3, 627-654, 782-783.

[GM1] G. Georgiev, O. Mathieu, Fusion rings for modular representations of Chevalley groups, Mathematical aspects of conformal and topological field theories and quantum groups (South Hadley, MA, 1992), 89-100, Contemp. Math., 175, Amer. Math. Soc., Providence, RI, 1994.

[GM2] G. Georgiev, O. Mathieu, Catégorie de fusion pour les groupes de Chevalley. [Fusion categories for Chevalley groups], C. R. Acad. Sci. Paris Sr. I Math. 315 (1992), no. 6, 659-662.

[GN] S. Gelaki and D. Naidu, Some properties of group-theoretical categories, J. Algebra 322 (2009), no. 8, 2631-2641.

[It] N. Ito, On the degrees of irreducible representations of a finite group, Nagoya Math. J. 3 (1951), pp. 5-6.

[Iz] M. Izumi, The structure of sectors associated with Longo-Rehren inclusions. II. Examples. Rev. Math. Phys. 13 (2001), no. 5, 603-674.

[Mi] G. O. Michler, Brauer's conjectures and the classification of finite simple groups, Representation Theory II, Groups and Orders, Lecture Notes in Math., Springer, Heidelberg (1986), pp. 129-142.

$[\mathrm{Mu}] \quad$ M. Müger, From subfactors to categories and topology. I. Frobenius algebras in and Morita equivalence of tensor categories, J. Pure Appl. Algebra 180 (2003), no. 1-2, 81-157.

[MS] S. Morrison and N. Snyder, Non-cyclotomic fusion categories, arXiv:1002.0168,

[N] D. Naidu, Categorical Morita equivalence for group-theoretical categories, Comm. Algebra 35 (2007), no. 11, 3544-3565.

[O] V. Ostrik, Module categories over the Drinfeld double of a finite group, Int. Math. Res. Not., 2003, no. 27, 1507-1520.

Department of Mathematics, Massachusetts Institute of Technology, CAMBridge, MA 02139, USA

E-mail address: etingof@math.mit.edu

Department of Mathematics, Technion-Israel Institute of TechnolOGY, HAIFA 32000, ISRAEL

E-mail address: gelaki@math.technion.ac.il 